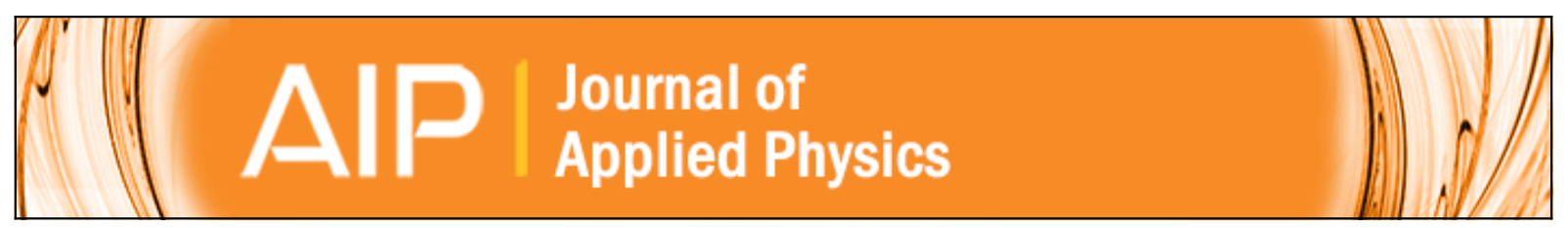

\title{
Vacancy complexes induce long-range ferromagnetism in GaN
}

Zhenkui Zhang, Udo Schwingenschlögl, and Iman S. Roqan

Citation: Journal of Applied Physics 116, 183905 (2014); doi: 10.1063/1.4901458

View online: http://dx.doi.org/10.1063/1.4901458

View Table of Contents: http://scitation.aip.org/content/aip/journal/jap/116/18?ver=pdfcov

Published by the AIP Publishing

\section{Articles you may be interested in}

Can cation vacancy defects induce room temperature ferromagnetism in GaN?

Appl. Phys. Lett. 102, 062411 (2013); 10.1063/1.4792528

Experimental evidence of Ga-vacancy induced room temperature ferromagnetic behavior in GaN films

Appl. Phys. Lett. 99, 162512 (2011); 10.1063/1.3654151

Effects of nitrogen vacancies on transition-metal-doped GaN: An ab initio study

J. Appl. Phys. 105, 103710 (2009); 10.1063/1.3132092

Ferromagnetic properties, electronic structure, and formation energy of Ga $0.9375 \mathrm{M} 0.0625 \mathrm{~N}$ ( M = vacancy,

Ca) by first principles study

J. Appl. Phys. 104, 043912 (2008); 10.1063/1.2970158

Manganese-induced long-range lattice disorder and vacancy formation in metal-organic chemical vapor deposition grown and ion-implanted Ga $1-x \mathrm{Mn} \times \mathrm{N}$

J. Vac. Sci. Technol. A 24, 1640 (2006); 10.1116/1.2201052

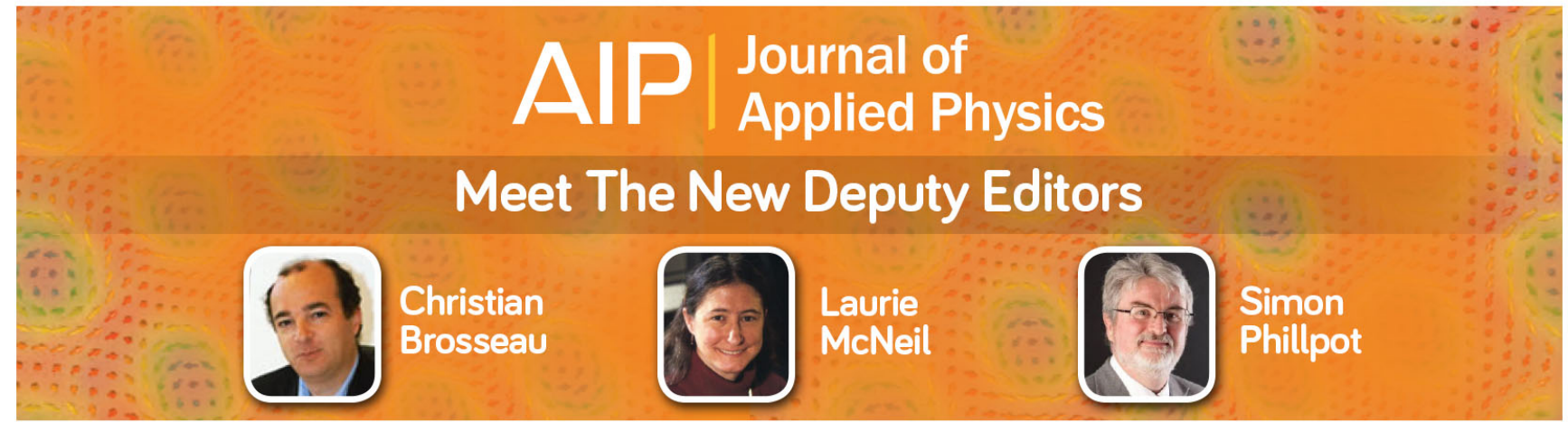




\title{
Vacancy complexes induce long-range ferromagnetism in GaN
}

\author{
Zhenkui Zhang, Udo Schwingenschlögl, ${ }^{\text {a) }}$ and Iman S. Roqan ${ }^{\text {a) }}$ \\ Physical Sciences and Engineering Division, King Abdullah University of Science and Technology (KAUST), \\ Thuwal 23955-6900, Kingdom of Saudi Arabia
}

(Received 7 October 2014; accepted 29 October 2014; published online 12 November 2014)

\begin{abstract}
By means of density functional theory, we argue that ferromagnetism in GaN can be induced by vacancy complexes. Spin polarization originates from the charge compensation between neutral $\mathrm{N}$ and Ga vacancies. Defect formation energy calculations predict that a vacancy complex of two positively charged $\mathrm{N}$ vacancies and one doubly negative Ga vacancy is likely to form. This defect complex induces a net moment of $1 \mu_{\mathrm{B}}$, which is localized around the negative Ga center and exhibits pronounced in-plane ferromagnetic coupling. In contrast to simple Ga vacancy induced ferromagnetism, the proposed picture is in line with the fact that $\mathrm{N}$ vacancies have a low formation energy. Formation energies indicate mutual stabilization of the intrinsic defects in GaN. (C) 2014 AIP Publishing LLC. [http://dx.doi.org/10.1063/1.4901458]
\end{abstract}

\section{INTRODUCTION}

After the prediction that GaN can become a room temperature ferromagnetic (FM) semiconductor, ${ }^{1}$ a large number of experimental efforts have been devoted to achieve diluted ferromagnetic GaN by introducing $3 d$ ions, ${ }^{2} 4 f$ ions, ${ }^{3,4}$ and $p$ defects. ${ }^{5,6}$ A special case is Gd-doped GaN, for which colossal magnetic moments have been reported. ${ }^{3}$ Subsequent studies found the $\mathrm{Gd}$ atoms to behave paramagnetically and showed only weak indications of moment formation for the Ga atoms. ${ }^{4,7}$ Complexes of $\mathrm{Gd}$ with $\mathrm{Ga}$ vacancies have been predicted to support ferromagnetism, ${ }^{8}$ similar to complexes of Gd with $\mathrm{N}$ and $\mathrm{O}$ interstitials. ${ }^{9}$ Though defect complexes are likely to be related to the observed magnetic behavior, ${ }^{10}$ understanding the exact mechanism remains a challenge.

Room temperature ferromagnetism through $p$ impurities and defect engineering has been realized in Refs. 11 and 12. In these studies, the $p$ defects, such as $\mathrm{Zn}$ vacancies, lead to local moments and mediate the exchange interaction. In this context, a picture of $\mathrm{Ga}$ vacancy $\left(\mathrm{V}_{\mathrm{Ga}}\right)$ induced ferromagnetism was developed for $\mathrm{GaN}$ based on results from density functional theory (DFT), ${ }^{13}$ ignoring the lower formation energy of the $\mathrm{N}$ vacancy $\left(\mathrm{V}_{\mathrm{N}}\right) .{ }^{14}$ Experiments also have attributed the ferromagnetism in undoped $\mathrm{GaN}$ to $\mathrm{V}_{\mathrm{Ga}}$, but found $\mathrm{V}_{\mathrm{N}}$ to be unavoidable to be introduced. ${ }^{5}$ Besides magnetically active complexes involving $\mathrm{Ga}$ vacancies in pristine $\mathrm{GaN},{ }^{6}$ also $\mathrm{GaN}$ vacancies $\left(\mathrm{V}_{\mathrm{GaN}}\right)$ in $\mathrm{Mg}$ and $\mathrm{Er}$ doped $\mathrm{GaN}$ and $\mathrm{GaN}$ vacancy complexes have been confirmed. ${ }^{15,16}$ In this work, we will demonstrate long-range ferromagnetism induced by vacancy complexes in wurtzite $\mathrm{GaN}$, incorporating both $\mathrm{V}_{\mathrm{Ga}}$ and $\mathrm{V}_{\mathrm{N}}$, rather than the previously proposed $\mathrm{V}_{\mathrm{Ga}}$ induced ferromagnetism. The magnetic interaction is found to be pronounced in the $a b$-plane and rather weak in the $c$-direction.

\section{COMPUTATIONAL METHODOLOGY}

All calculations are performed using the VASP code ${ }^{17}$ with projector augmented wave pseudopotentials ${ }^{18}$ and an

\footnotetext{
a) Authors to whom correspondence should be addressed. Electronic addresses: Udo.Schwingenschlogl@kaust.edu.sa and Iman.Roqan@kaust.edu.sa
}

energy cutoff of $400 \mathrm{eV}$. The valence shells are $2 s^{2} 2 p^{3}$ for $\mathrm{N}$ and $3 d^{10} 4 s^{2} 4 p^{1}$ for $\mathrm{Ga}$, where the semicore $d$ states are explicitly included for Ga. The Perdew-Burke-Ernzerhof flavor of the generalized gradient approximation is employed, with an on-site Coulomb repulsion to account for electronic correlation effects. ${ }^{19}$ A value of $U_{\text {eff }}=5 \mathrm{eV}$ is adopted for both the Ga $d$ and $\mathrm{N} p$ states. The optimized lattice parameters ( $a=3.129 \AA, c / a=1.627$ ) of wurtzite $\mathrm{GaN}$ agree with the experimental values ( $a=3.19 \AA, c / a=1.627)$, while the calculated band gap of $2.88 \mathrm{eV}$ underestimates the experimental value of $3.44 \mathrm{eV}$. All defective structures are fully relaxed until the forces per atom have declined to less than $0.04 \mathrm{eV} / \AA$. The method of Gaussian smearing $($ ISMEAR $=0)$ was selected for electron occupations with a width of $0.08 \mathrm{eV}$ for 192-atom and its doubled supercells and $0.04 \mathrm{eV}$ for 300-atom supercells and its doubled supercells. An energy convergence of $5 \times 10^{-5} \mathrm{eV}$ is achieved. For 192 and 300-atom supercells $2 \times 2 \times 1 \mathrm{k}$-meshes are found to be sufficient, while denser $\mathrm{k}$-meshes are used for smaller supercells. For the 128-atom supercell the ion positions are allowed to relax together with the size and shape of the supercell. For the 192- and 300-atom supercells, we observe that the vacancy defects have a very slight effect on the lattice constant due to a very low concentration. Therefore, the ion positions are relaxed only as the size of supercell remains unchanged. The concerned magnetic properties are found to be independent of volume relaxation in the cases of 192- and 300-atom supercells.

\section{RESULTS AND DISCUSSION}

Supercells of different sizes containing one $\mathrm{V}_{\mathrm{GaN}}$ are found to converge to a spin-polarized ground state with a magnetic moment of $2 \mu_{B}$. The results in Table I refer either to an onsite repulsion on both the Ga $d$ and $\mathrm{N} p$ states $\left(U_{d, p}\right)$ or to one on the Ga $d$ states only $\left(U_{d}\right)$, which corrects the energetic position of the Ga $d$ states as well as the hybridization with the $\mathrm{N} p$ states to enhance the electronic structure description. ${ }^{20}$ Comparing the results obtained by the two 
TABLE I. Effects of the supercell size and onsite repulsion on the magnetic moments on the $\mathrm{N}\left(\mathrm{M}_{\mathrm{N}}\right)$ and $\mathrm{Ga}\left(\mathrm{M}_{\mathrm{Ga}}\right)$ neighbors of $\mathrm{V}_{\mathrm{GaN}} \cdot \mathrm{M}_{\mathrm{N}}$ and $\mathrm{M}_{\mathrm{Ga}}$ (in $\mu_{B}$ ) are obtained by extrapolating the sum of moments over all the atoms to the total $2 \mu_{B}$ moment. $\mathrm{M}_{\mathrm{N}(\mathrm{i})}(\mathrm{i}=1,2,3)$ indicates the local moment spreading on the first, second, and third nearest $\mathrm{N}$ neighbors of the $\mathrm{V}_{\mathrm{Ga}}$ center, respectively. $\mathrm{M}_{\mathrm{Ga}}$ indicates the local moment on the first Ga neighbors of the $\mathrm{V}_{\mathrm{N}}$ center. $\mathrm{E}_{\text {spin }}$ (in $\mathrm{meV}$ ) is the energy difference between the spin degenerate and the spin-polarized state, indicating the stability of spin polarization. $\mathrm{E}_{\mathrm{mag}}(\mathrm{in} \mathrm{meV}$ ) is the energy difference between AFM and FM states, calculated by doubling the supercells.

\begin{tabular}{|c|c|c|c|c|c|c|}
\hline & \multicolumn{2}{|c|}{128} & \multicolumn{2}{|c|}{192} & \multicolumn{2}{|c|}{300} \\
\hline & $U_{d}$ & $U_{d, p}$ & $U_{d}$ & $U_{d, p}$ & $U_{d}$ & $U_{d, p}$ \\
\hline $\mathrm{M}_{\mathrm{N}(1)}$ & 0.38 & 0.54 & 0.47 & 0.55 & 0.47 & 0.54 \\
\hline $\mathrm{M}_{\mathrm{N}(2)}$ & 0.04 & 0.04 & 0.04 & 0.04 & 0.04 & 0.04 \\
\hline $\mathrm{M}_{\mathrm{N}(2)}$ & & & & & 0.00 & 0.00 \\
\hline $\mathrm{M}_{\mathrm{Ga}}$ & 0.05 & 0.01 & 0.01 & 0.00 & 0.01 & 0.00 \\
\hline $\mathrm{E}_{\text {spin }}$ & 273 & 818 & 328 & 802 & 318 & 825 \\
\hline$E_{m a g}$ & 92 & -9 & -28 & -6 & -10 & -2 \\
\hline
\end{tabular}

models $\left(U_{d, p}\right.$ and $\left.U_{d}\right)$ allows us to check whether electron correlations in the $\mathrm{N} p$ states are important (so far, an open question $^{21,22}$ ). The results for the $U_{d}$ model with a 128-atom supercell show differences to those for the $\mathrm{U}_{d, p}$ model, which gives rise to consistent values for all supercell sizes (indicating that in this case the defect states are more localized). We conclude that the 192-atom supercell is sufficiently large to describe a $\mathrm{V}_{\mathrm{GaN}}$. The local moment extends to the second nearest $\mathrm{N}$ neighbors, as for the 300-atom supercell we obtain a negligible moment on the third nearest neighbors.

We have studied various configurations with different distances of the $\mathrm{V}_{\mathrm{Ga}}$ and $\mathrm{V}_{\mathrm{N}}$ and find a moment of $2 \mu_{B}$ for every case except for two situations that have the Ga and $\mathrm{N}$ dangling bonds overlap directly. In these cases, the absence of spin polarization is a result of electron-hole compensation by the bonding between the dangling states of $\mathrm{Ga}$ and $\mathrm{N}$. This bonding is accompanied by enhanced distortions. It is also found that $\mathrm{V}_{\mathrm{GaN}}\left(\mathrm{V}_{\mathrm{Ga}}\right.$ and $\mathrm{V}_{\mathrm{N}}$ are nearest neighbors) is energetically favorable over configurations with larger separation. The binding energy needed to form $\mathrm{V}_{\mathrm{GaN}}$ from $\mathrm{V}_{\mathrm{Ga}}$ and $\mathrm{V}_{\mathrm{N}}$ in neutral states is $2.3 \mathrm{eV}$, indicating a strong tendency of aggregation.

To demonstrate the origin of the $2 \mu_{B}$ magnetic moment, we schematically present the electronic structures of isolated $\mathrm{V}_{\mathrm{Ga}}$ and $\mathrm{V}_{\mathrm{N}}$ (Fig. 1), based on our calculations that are in qualitative agreement with previous DFT studies. ${ }^{14,23,24} \mathrm{~A}$ neutral $\mathrm{V}_{\mathrm{Ga}}$ extracts three electrons from the direct $\mathrm{N}$ neighbors and gives rise to $a_{l}$ and triplet-like defect states, which hereafter are referred to as $t$ states. The spin-up $t$ states are filled and are part of the valence band, whereas the spindown $t$ states are empty (Fig. 1(a)). A neutral $\mathrm{V}_{\mathrm{N}}$ releases three electrons to its Ga neighbors. Two of them form a filled $a_{l}^{\prime}$ state, which is located within the valence band, whereas the remaining electron occupies $t^{\prime}$ states next to the conduction band (Fig. 1(b)). ${ }^{23}$ Consequently, when $V_{\mathrm{Ga}}$ interacts with $V_{N}$ this high-energy electron from $V_{N}$ is released to $\mathrm{V}_{\mathrm{Ga}}$, forming a $\mathrm{V}_{\mathrm{Ga}}{ }^{-}-\mathrm{V}_{\mathrm{N}}{ }^{+}$pair, leading to the net moment

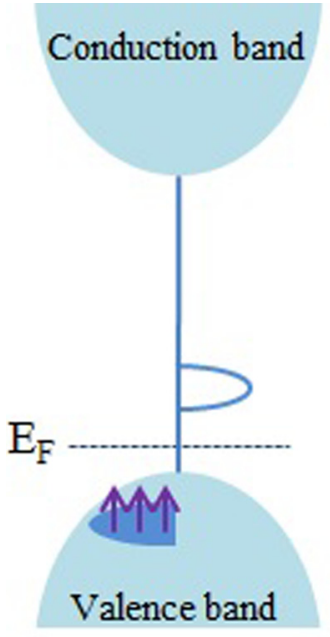

(a) $\mathrm{V}_{\mathrm{Ga}}$

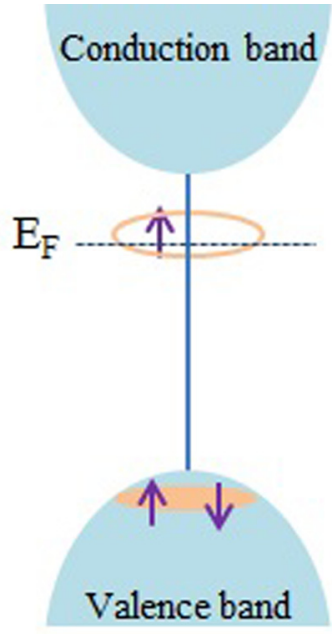

(b) $\mathrm{V}_{\mathrm{N}}$
FIG. 1. Energy diagram of defective GaN containing an isolated (a) $\mathrm{V}_{\mathrm{Ga}}$ and (b) $\mathrm{V}_{\mathrm{N}}$. The left (right) part refers to the spin-up (spin-down) channel.

of $2 \mu_{B}$. This charge transfer agrees with the argument that $\mathrm{V}_{\mathrm{N}}{ }^{+}$is the energetically most relevant defect. ${ }^{13,23,25}$

For $\mathrm{V}_{\mathrm{GaN}}$ and separated $\mathrm{V}_{\mathrm{Ga}}$ and $\mathrm{V}_{\mathrm{N}}$ (separation $8.5 \AA$ ), the total density of states (DOS) and the DOS projected on the $\mathrm{N}$ and $\mathrm{Ga}$ nearest neighbors are plotted in Fig. 2 (192-atom supercell). For $\mathrm{V}_{\mathrm{GaN}}$ the insulating character is maintained with a reduced band gap of $0.53 \mathrm{eV}$. The in-gap states are due to the $\mathrm{N}$ nearest neighbors (mainly $p_{x}+p_{y}$ ) and are spin polarized. The Ga neighbors contribute to the defect states near the conduction band minimum (CBM), see Fig. 2, where hybridization of the Ga $p$ and $\mathrm{N} p$ states appears at the valence band maximum (VBM). On the other hand, for separated $\mathrm{V}_{\mathrm{Ga}}$ and $\mathrm{V}_{\mathrm{N}}$, we always find a half-metallic character. Changing $U$ from 3 to $7 \mathrm{eV}$ on the $\mathrm{N} p$ states has essentially no influence on the electronic structures.

Fig. 3(a) illustrates the spatial extension of the two holes trapped at the $\mathrm{N}$ neighbors of $\mathrm{V}_{\mathrm{GaN}}$, mainly in the $a b$-plane (three direct $\mathrm{N}$ neighbors). The first $\mathrm{N}$ neighbors of the $\mathrm{V}_{\mathrm{Ga}}$ center exhibit displacement away from the vacancy center, while the $\mathrm{Ga}$ neighbors of the $\mathrm{V}_{\mathrm{N}}$ side show displacement approaching the vacancy center. Similar behaviors are also found in the separated vacancy complexes, as will be demonstrated below. Fig. 3(b) shows that the distribution of the separated vacancies (distance between the centers of $\mathrm{V}_{\mathrm{Ga}}{ }^{-}$ and $\mathrm{V}_{\mathrm{N}}{ }^{+}$is $8.5 \AA$ ) remains predominantly planar around the $\mathrm{V}_{\mathrm{Ga}}$, with moments of $0.46 \mu_{B}$ on each of the three in-plane $\mathrm{N}$ neighbors, despite a significant spin density on the single out-of-plane $\mathrm{N}$ neighbor $\left(0.26 \mu_{B}\right)$. The $\mathrm{V}_{\mathrm{N}}$ does not trap holes. Fig. 3(c) is analogous to Fig. 3(b) but for the case of a single hole, i.e., an additional electron is introduced. The inplane character is very pronounced and the bonds to the three in-plane $\mathrm{N}$ neighbors are elongated $(1.821 \AA)$ to even exceed that of the out-of-plane neighbor $(1.806 \AA)$. In Fig. 3(d) we address a single hole trapped in a $\mathrm{V}_{\mathrm{Ga}}-2 \mathrm{~V}_{\mathrm{N}}$ complex, again showing the pronounced in-plane character. The distance between $\mathrm{V}_{\mathrm{Ga}}{ }^{-}$and the second $\mathrm{V}_{\mathrm{N}}{ }^{+}$is $7.6 \AA$ in Fig. $3(\mathrm{~d})$, whereas that between the two $\mathrm{V}_{\mathrm{N}}{ }^{-}$centers is $5.4 \AA$. The 

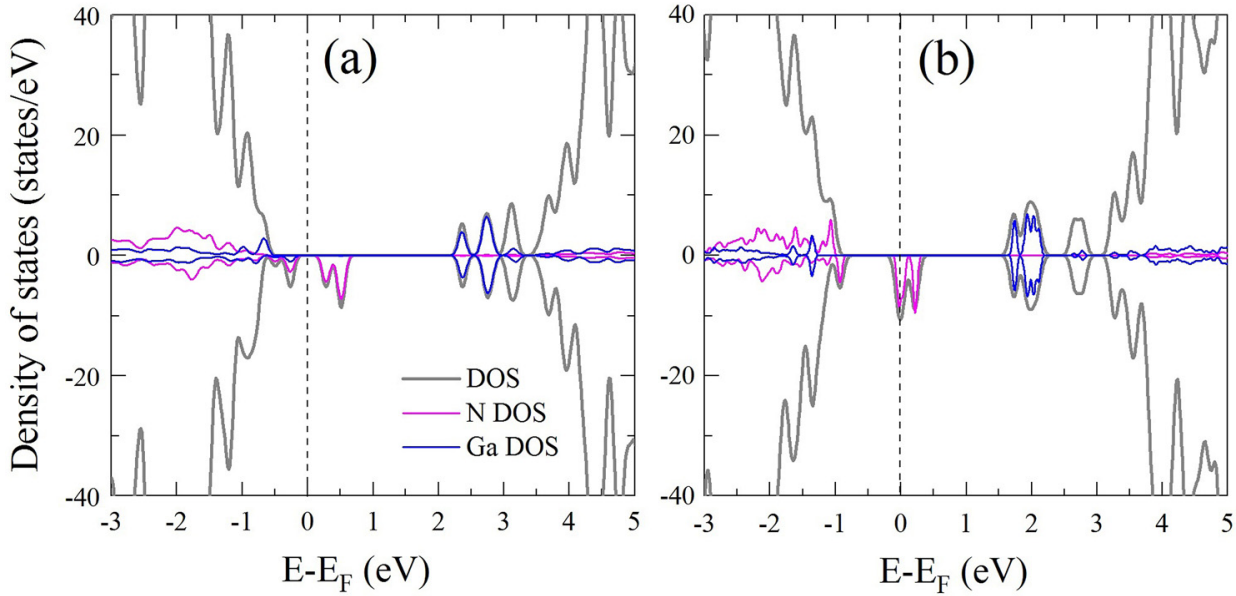

FIG. 2. Total and partial DOS of a (a) $\mathrm{V}_{\mathrm{GaN}}$ and (b) $\mathrm{V}_{\mathrm{Ga}}{ }^{-}-\mathrm{V}_{\mathrm{N}}{ }^{+}$complex. The partial DOS refers to the $\mathrm{N}$ (magenta) and Ga (blue) neighbors of the defect. discussed in-plane distribution of the holes is distinct from the isotropic distribution reported for zinc-blende $\mathrm{GaN},{ }^{13}$ showing that the magnetic interaction is much more anisotropic in the present wurtzite case.

We calculate the total energies for antiferromagnetic (AFM) and FM ordering between neighboring complexes in various charge states by doubling the 192 and 300-atom supercells along the $a$-axis. Table II gives the energy differences $\mathrm{E}_{\mathrm{mag}}=\mathrm{E}_{\mathrm{AFM}}-\mathrm{E}_{\mathrm{FM}}$ and the magnetic moments. The FM ordering is found to be favorable for a distance of $12.4 \AA$, except for neutral $\mathrm{V}_{\mathrm{GaN}}$ and $\mathrm{V}_{\mathrm{Ga}}$, and is likely to survive at room temperature (about $23.5 \mathrm{meV}$ (Ref. 26)), given the large energetic preference $\left(\mathrm{E}_{\mathrm{mag}}\right)$. The ordering of two neutral $\mathrm{V}_{\mathrm{Ga}}$ can only be AFM, because the $t$ orbital is occupied by three electrons with parallel spin (see Fig. 1(a)). The same picture applies to neutral $\mathrm{V}_{\mathrm{GaN}}$, because the states around the Fermi energy $\left(E_{F}\right)$ in Fig. 2(a) are fully spin polarized. Charging $\mathrm{V}_{\mathrm{Ga}}$ by either introducing an additional electron or creating $\mathrm{V}_{\mathrm{N}}$ shifts $E_{F}$ upwards in energy to partially occupy the defect states (see Fig. 2(b)). In this case, FM alignment between the local moments of neighboring complexes is possible (see Table II). This argument is also valid for negatively charged $V_{\mathrm{GaN}}$, for which $\mathrm{E}_{\mathrm{mag}}$ is larger than found in previous DFT studies of zinc-blende bulk and the surface of wurtzite GaN. ${ }^{13,27}$ It is noted that the absolute value of $\mathrm{E}_{\mathrm{mag}}$ in the $U_{d}$ model is considerably larger than that in the $U_{d, p}$ model (see Table I), demonstrating the role of electronic correlations in the $\mathrm{N} p$ orbital.

The magnetic interaction is found to be substantial at a separation of $12.5 \AA$ between two $\mathrm{V}_{\mathrm{Ga}}{ }^{-}-\mathrm{V}_{\mathrm{N}}{ }^{+}$complexes, but it declines quickly at a separation of $15.5 \AA$ (see Table II). This picture is confirmed by the spin density maps in Fig. 4, which show overlap between the two defects at a separation of $12.5 \AA$ but not at $15.5 \AA$. An additional configuration
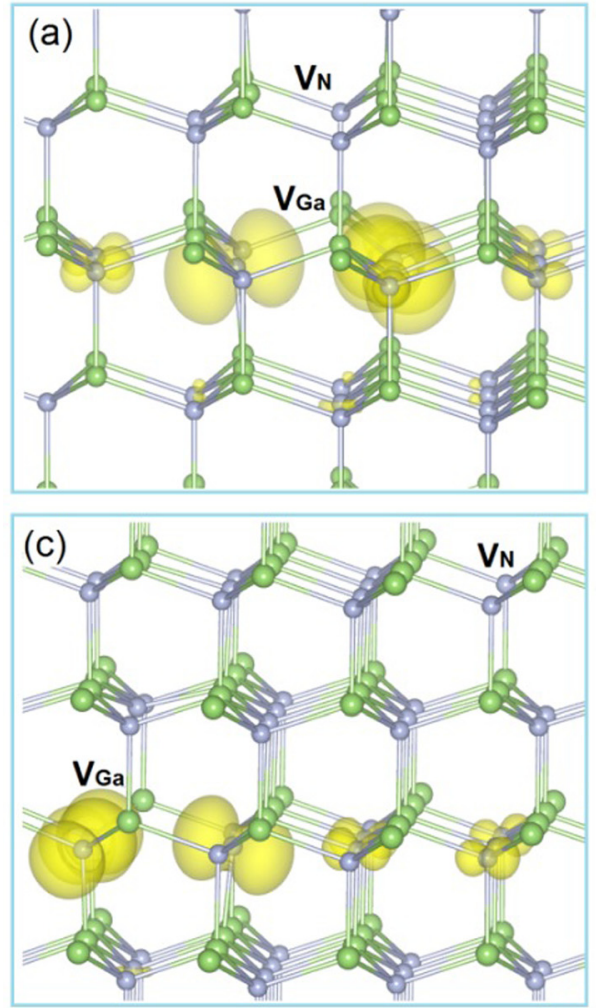
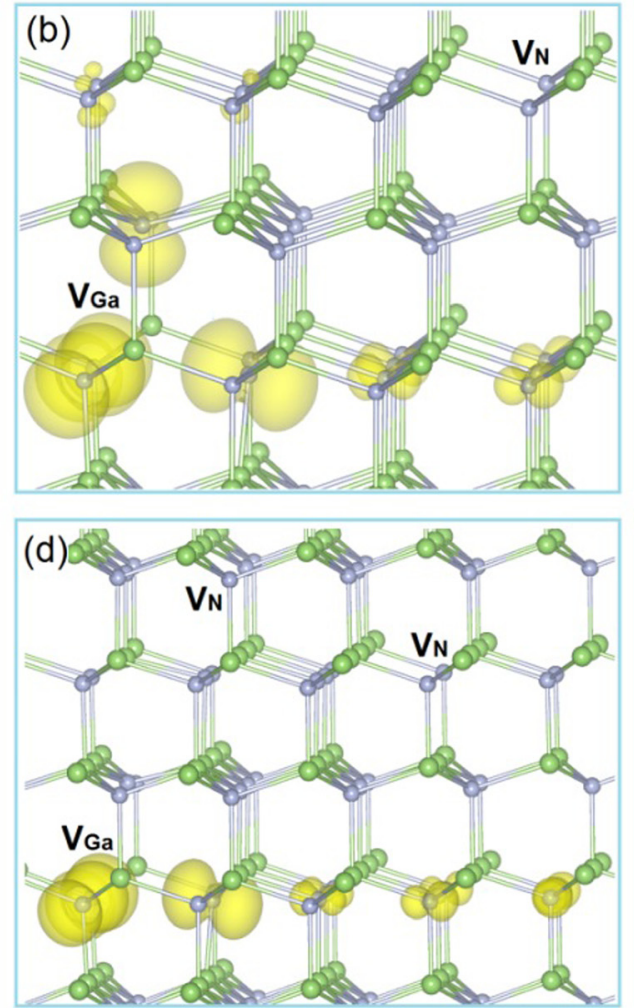

FIG. 3. Spin density of (a) a neutral $\mathrm{V}_{\mathrm{GaN}}(2$ holes $)$, (b) a neutral $\mathrm{V}_{\mathrm{Ga}}{ }^{-}-\mathrm{V}_{\mathrm{N}}{ }^{+}$complex (2 holes), (c) a negatively charged $\mathrm{V}_{\mathrm{Ga}}{ }^{2-}-\mathrm{V}_{\mathrm{N}}{ }^{+}$complex (1 hole), and (d) a neutral $\mathrm{V}_{\mathrm{Ga}}{ }^{2-}-2 \mathrm{~V}_{\mathrm{N}}{ }^{+}$complex (1 hole). The isosurface level is $0.002 e / \AA^{3}$. Larger and smaller spheres represent the Ga and $\mathrm{N}$ atoms, respectively. 
TABLE II. Total magnetic moment $\left(\mathrm{M}_{\mathrm{tot}}\right)$ and $\mathrm{E}_{\mathrm{mag}}$ (for two separations $d$ ) in various charge states. The moments are given in $\mu_{B}$, the energies in meV, and the separations in $\AA$. The $d$ indicates the distance between two $\mathrm{V}_{\mathrm{Ga}}$ centers arising from two vacancy complexes, while the distance between the two $V_{N}$ centers is ignored as there is no spin polarization surrounding their centers. For the complex $\mathrm{V}_{\mathrm{Ga}}{ }^{-}-\mathrm{V}_{\mathrm{N}}{ }^{+}$, the distance between $\mathrm{V}_{\mathrm{Ga}}{ }^{-}$and $\mathrm{V}_{\mathrm{N}}{ }^{+}$ is $8.5 \AA$. For the $\mathrm{V}_{\mathrm{Ga}}{ }^{2-}-2 \mathrm{~V}_{\mathrm{N}}{ }^{+}$complex, the distance between $\mathrm{V}_{\mathrm{Ga}}{ }^{2-}$ and the second $\mathrm{V}_{\mathrm{N}}{ }^{+}$is $7.6 \AA$, whereas that between two $\mathrm{V}_{\mathrm{N}}{ }^{-}$centers is $5.4 \AA$.

\begin{tabular}{lcccc}
\hline \hline & & & \multicolumn{2}{c}{$\mathrm{E}_{\text {mag }}$} \\
\cline { 3 - 5 } Defect & Charge & $\mathrm{M}_{\text {tot }}$ & $d=12.4 \AA$ & $d=15.5 \AA$ \\
\hline $\mathrm{V}_{\mathrm{GaN}}$ & 0 & 2.0 & -6 & -2 \\
$\mathrm{~V}_{\mathrm{Ga}}{ }^{-}-\mathrm{V}_{\mathrm{N}}{ }^{+}$ & -1 & 1.0 & 87 & 5 \\
$\mathrm{~V}_{\mathrm{Ga}}{ }^{2-}-2 \mathrm{~V}_{\mathrm{N}}{ }^{+}$ & 0 & 2.0 & 67 & -1 \\
$\mathrm{~V}_{\mathrm{Ga}}$ & -1 & 1.0 & 70 & 14 \\
& 0 & 1.0 & 57 & 11 \\
& 0 & 3.0 & -8 & -1 \\
& -1 & 2.0 & 127 & -1 \\
\hline \hline
\end{tabular}

has been examined with the two $\mathrm{V}_{\mathrm{Ga}}$ in adjacent Ga layers in a distance of $11.2 \AA$. In this case, the FM and AFM states are energetically degenerate, suggesting paramagnetism, due to negligible overlap of the spin of the defect states. A dominantly in-plane magnetic coupling agrees with the anisotropic character of the magnetism in wurtzite $\mathrm{ZnO}$ after carbon-doping. ${ }^{28}$

Fig. 5 gives formation energies for $\mathrm{V}_{\mathrm{GaN}}, \mathrm{V}_{\mathrm{Ga}}$, and $\mathrm{V}_{\mathrm{N}}$ as a function of the Fermi energy, using $\mathrm{E}_{\text {form }}\left[\mathrm{D}^{q}\right]=E_{\text {tot }}$ $\left[\mathrm{D}^{q}\right]-E_{\mathrm{tot}}+\sum n_{i} \mu_{i}+q\left[E_{F}+E_{\mathrm{V}}\right],{ }^{29}$ where $E_{\mathrm{tot}}$ and $E_{\mathrm{tot}}$ $\left[\mathrm{D}^{q}\right]$ are the total energies of the non-defective and defective (with charge $q$ ) supercells, respectively, $n_{i}$ denotes the number of atoms introduced to $\left(n_{i}<0\right)$ or subtracted from $\left(n_{i}>0\right)$ the pristine supercell, $\mu_{i}$ is the chemical potential, and $E_{F}$ denotes the Fermi energy (measured with respect to the pristine VBM, varying from zero to the experimental band gap). The chemical potentials, $\mu_{\mathrm{Ga}}(\mathrm{Ga}-\mathrm{rich} / \mathrm{N}-$ poor $)$ and $\mu_{\mathrm{N}}(\mathrm{N}$-rich/Ga-poor), are calculated from metallic $\mathrm{Ga}$ and the $\mathrm{N}_{2}$ molecule, respectively. In Ga-poor/N-rich environment, for example, $\mu_{\mathrm{Ga}}=\mu_{\mathrm{GaN}}-\mu_{\mathrm{N}}$, where $\mu_{\mathrm{GaN}}$ is given by the energy of a formula unit of $\mathrm{GaN}$ and $\mu_{\mathrm{N}}$ by half of the energy of a $\mathrm{N}_{2}$ molecule. According to Fig. $5(\mathrm{a}), \mathrm{V}_{\mathrm{N}}$ has $3+$, $1+$, and 0 states as $E_{F}$ varies from the VBM to the CBM, whereas the $2+$ state is unstable, behaving as a negative- $U$ defect. These results and the value of $E_{\text {form }}$ at the VBM $(-1.14 / 0.31 \mathrm{eV}$ in $\mathrm{N}$-poor/N-rich environment) agree with a recent hybrid functional study. ${ }^{29} \mathrm{~V}_{\mathrm{GaN}}$ has 0,1 -, and 2states, consistent with Ref. 14, where $\mathrm{E}_{\text {form }}$ falls in between that of $\mathrm{V}_{\mathrm{Ga}}$ in Ga-rich and Ga-poor environments. $\mathrm{E}_{\text {form }}$ does not depend on $\mu_{\mathrm{Ga}}$ or $\mu_{\mathrm{N}}$, but on $\mu_{\mathrm{GaN}}$, as a formula unit of $\mathrm{GaN}$ is removed. The small stability range of the 1-state reduces the possibility that ferromagnetism is induced by $\mathrm{V}_{\mathrm{GaN}}$.

$\mathrm{V}_{\mathrm{Ga}}$ can have charge states 0 and 2-, whereas the 1-state is metastable and the 3 -state is unstable. This result contradicts previous studies (finding the existence of $0,-1,-2$, and -3 states) in which the onsite Coulomb interaction of the Ga $d$ states was not taken into account. ${ }^{14,30}$ To verify its influence, we have calculated $\mathrm{E}_{\text {form }}$ of $\mathrm{V}_{\mathrm{Ga}}$ using the $U_{d}$ model with $U_{\text {eff }}=5 \mathrm{eV}$ to Ga $d$ states, see Fig. 5(b), confirming a 2- state over a wide energy range and an unstable 3state. Interestingly, plain GGA calculations using the $U_{d, p}$ converged structures qualitatively reproduce the wrong trend of Refs. 14 and 30 (see Fig. 5(c)). We therefore conclude convincingly that the discrepancy arises from electronic correlations in the $\mathrm{Ga} d$ orbitals. The experimental observation ${ }^{31}$ showed that negatively charged Ga vacancy of 2- state is more stable than that of 3- state, which supports our finding that this state is stable. The stability of $\mathrm{V}_{\mathrm{N}}^{+}$and $\mathrm{V}_{\mathrm{Ga}}{ }^{2-}$ let $\mathrm{us}$ expect ferromagnetism in $\mathrm{GaN}$ when the ratio of $\mathrm{V}_{\mathrm{Ga}}$ to $\mathrm{V}_{\mathrm{N}}$ approaches 0.5 , because $\mathrm{V}_{\mathrm{Ga}}{ }^{2-}$ captures two electrons from two $\mathrm{V}_{\mathrm{N}}$ (leaving $\mathrm{V}_{\mathrm{N}}{ }^{+}$). During this process, $\mathrm{V}_{\mathrm{Ga}}$ and $\mathrm{V}_{\mathrm{N}}$ are mutually stabilized with respect to their neutral states. As a consequence, long-range in-plane ferromagnetism is formed
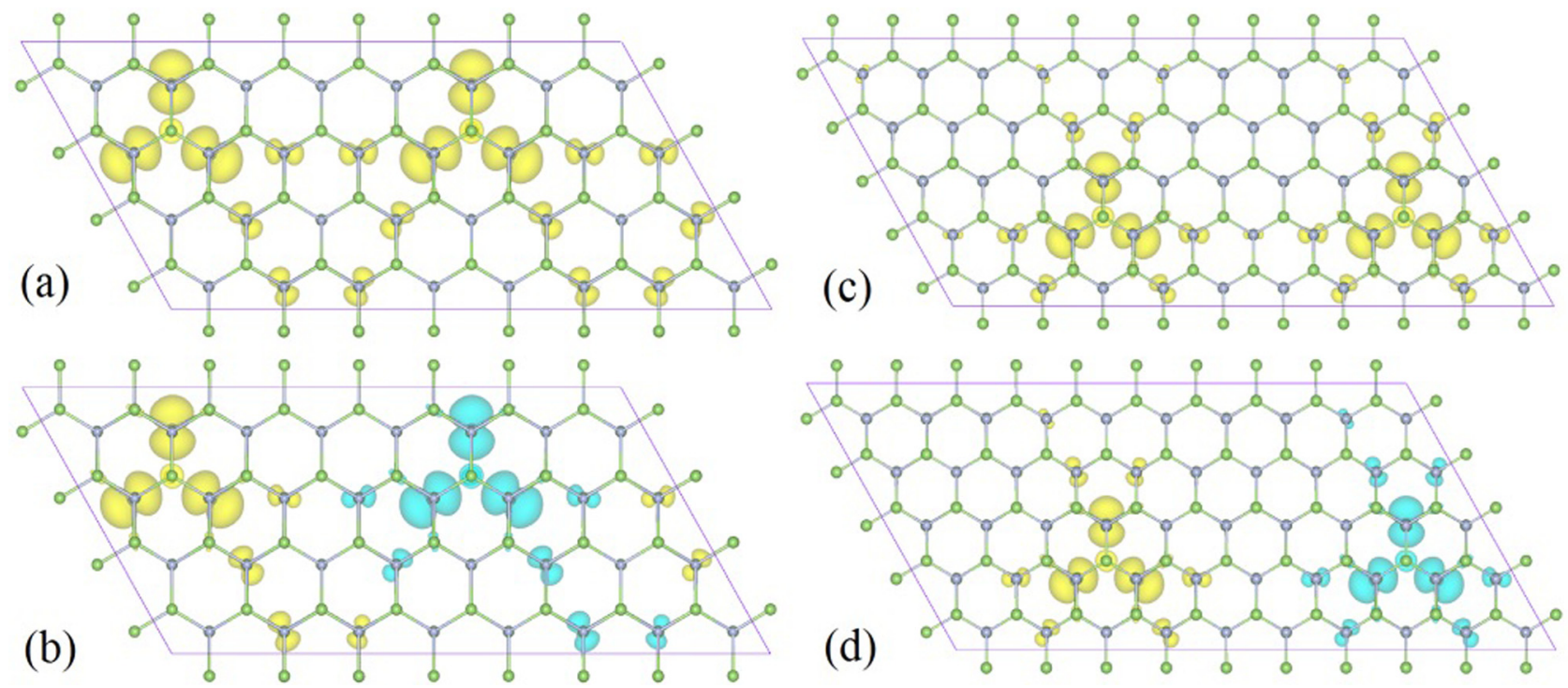

FIG. 4. Spin density for ((a) and (c)) FM and ((b) and (d)) AFM ordering between two $\mathrm{V}_{\mathrm{Ga}}{ }^{2-}-\mathrm{V}_{\mathrm{N}}{ }^{+}$complexes with one trapped hole. Yellow (blue) refers to spin-up (spin-down), for an isosurface level of $0.001 e / \AA^{3}$. 

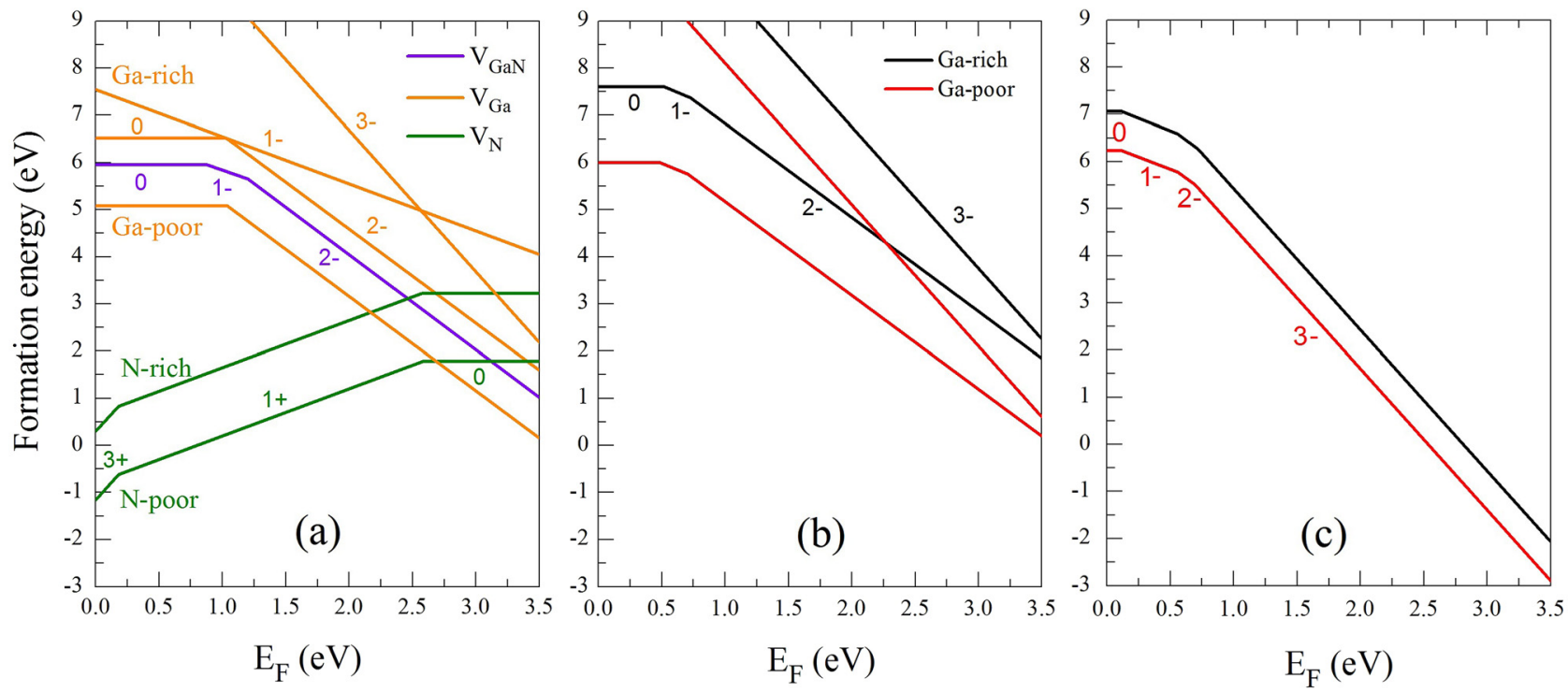

FIG. 5. (a) Formation energies of $\mathrm{V}_{\mathrm{GaN}}, \mathrm{V}_{\mathrm{Ga}}$, and $\mathrm{V}_{\mathrm{N}}$ in various charge states as functions of the Fermi energy, which is calculated using the $U_{d, p}$ model, namely, applying the onsite repulsion $U$ to both $\mathrm{N} p$ and Ga $d$ states $\left(U_{\mathrm{eff}}=5 \mathrm{eV}\right.$ for the $\mathrm{N} p$ and Ga $d$ states). The results of the formation energy of $\mathrm{V}_{\mathrm{Ga}}$ calculated for (b) the onsite repulsion are only applied to Ga $d$ states $\left(U_{\text {eff }}=5 \mathrm{eV}\right)$ and (c) for plain GGA-PBE, without onsite repulsion on either $\mathrm{N} p$ or Ga $d$ states.

between the Ga defects in a wide range of $1.0 \leq E_{F} \leq 2.5 \mathrm{eV}$ (see Fig. 5(a)).

In conclusion, we predict long-range ferromagnetism induced by vacancy complexes in GaN at distances up to about $13 \AA$. The distance corresponds to a vacancy complex concentration of about $1 \%$, which is a realistic amount. The ferromagnetism turns out to be of remarkably strong in-plane character. The spin polarization originates from the charge compensation between neutral $\mathrm{V}_{\mathrm{N}}{ }^{0}$ and $\mathrm{V}_{\mathrm{Ga}}{ }^{0}$ forming a complex that consists of two $\mathrm{V}_{\mathrm{N}}{ }^{+}$and one $\mathrm{V}_{\mathrm{Ga}}{ }^{2-}$ with a net moment of $1 \mu_{B}$ localized on $\mathrm{V}_{\mathrm{Ga}}{ }^{2-}$. This charge transfer leads to a sharp mutual stabilization of the cation and anion vacancies according to the calculated defect formation energies. For example, $\mathrm{E}_{\text {form }}\left[\mathrm{V}_{\mathrm{Ga}}{ }^{2-}\right]+2 \mathrm{E}_{\text {form }}\left[\mathrm{V}_{\mathrm{N}}{ }^{+}\right]-$ $\mathrm{E}_{\text {form }}\left[\mathrm{V}_{\mathrm{Ga}}{ }^{0}\right]-2 \mathrm{E}_{\text {form }}\left[\mathrm{V}_{\mathrm{N}}{ }^{0}\right]=3 \mathrm{eV}$ for $E_{F}=1 \mathrm{eV}$ in the $\mathrm{N}$-rich environment. In fact, $\mathrm{V}_{\mathrm{N}}{ }^{+}$and $\mathrm{V}_{\mathrm{Ga}}{ }^{2-}$ are the most relevant defects over a large range of $E_{F}$. Our findings provide a new understanding of the role of intrinsic defects in the ferromagnetism in $\mathrm{GaN}$.

${ }^{1}$ T. Dietl, H. Ohno, F. Matsukura, J. Cibert, and D. Ferrand, Science 287, 1019 (2000).

${ }^{2}$ N. Theodoropoulou, A. F. Hebard, M. E. Overberg, C. R. Abernathy, S. J. Pearton, S. N. G. Chu, and R. G. Wilson, Appl. Phys. Lett. 78, 3475 (2001).

${ }^{3}$ S. Dhar, O. Brandt, M. Ramsteiner, V. F. Sapega, and K. H. Ploog, Phys. Rev. Lett. 94, 037205 (2005).

${ }^{4}$ A. Ney, T. Kammermeier, V. Ney, S. Ye, K. Ollefs, E. Manuel, S. Dhar, K. H. Ploog, E. Arenholz, F. Wilhelm, and A. Rogalev, Phys. Rev. B 77, 233308 (2008).

${ }^{5}$ C. Madhu, A. Sundaresan, and C. N. R. Rao, Phys. Rev. B 77, 201306(R) (2008).

${ }^{6}$ L. Kilanski, F. Tuomisto, R. Szymczak, and R. Kruszka, Appl. Phys. Lett. 101, 072102 (2012).

${ }^{7}$ L. Liu, P. Y. Yu, Z. Ma, and S. S. Mao, Phys. Rev. Lett. 100, 127203 (2008).

${ }^{8}$ Y. Gohda and A. Oshiyama, Phys. Rev. B 78, 161201(R) (2008).
${ }^{9}$ C. Mitra and W. R. L. Lambrecht, Phys. Rev. B 80, 081202(R) (2009).

${ }^{10}$ M. Roever, J. Malindretos, A. Bedoya-Pinto, A. Rizzi, C. Rauch, and F. Tuomisto, Phys. Rev. B 84, 081201(R) (2011).

${ }^{11}$ H. Pan, J. B. Yi, L. Shen, R. Q. Wu, J. H. Yang, J. Y. Lin, Y. P. Feng, J. Ding, L. H. Van, and J. H. Yin, Phys. Rev. Lett. 99, 127201 (2007).

${ }^{12}$ J. B. Yi, C. C. Lim, G. Z. Xing, H. M. Fan, L. H. Van, S. L. Huang, K. S. Yang, X. L. Huang, X. B. Qin, B. Y. Wang, T. Wu, L. Wang, H. T. Zhang, X. Y. Gao, T. Liu, A. T. S. Wee, Y. P. Feng, and J. Ding, Phys. Rev. Lett. 104, 137201 (2010).

${ }^{13}$ P. Dev, Y. Xue, and P. Zhang, Phys. Rev. Lett. 100, 117204 (2008).

${ }^{14}$ M. G. Ganchenkova and R. M. Nieminen, Phys. Rev. Lett. 96, 196402 (2006).

${ }^{15}$ A. Uedono, S. Ishibashi, K. Tenjinbayashi, T. Tsutsui, K. Nakahara, D. Takamizu, and S. F. Chichibu, J. Appl. Phys. 111, 014508 (2012).

${ }^{16}$ S. Chen, A. Uedono, S. Ishibashi, S. Tomita, H. Kudo, and K. Akimoto, Appl. Phys. Lett. 96, 051907 (2010).

${ }^{17}$ G. Kresse and D. Joubert, Phys. Rev. B 59, 1758 (1999).

${ }^{18}$ P. E. Blöchl, Phys. Rev. B 50, 17953 (1994).

${ }^{19}$ S. L. Dudarev, G. A. Botton, S. Y. Savrasov, C. J. Humphreys, and A. P. Sutton, Phys. Rev. B 57, 1505 (1998).

${ }^{20}$ A. Janotti, D. Segev, and C. G. van de Walle, Phys. Rev. B 74, 045202 (2006).

${ }^{21}$ V. Pardo and W. E. Pickett, Phys. Rev. B 78, 134427 (2008).

${ }^{22}$ O. Volnianska and P. Boguslawski, J. Phys.: Condens. Matter 22, 073202 (2010).

${ }^{23}$ J. Neugebauer and C. G. van de Walle, Phys. Rev. B 50, 8067 (1994).

${ }^{24}$ O. Volnianska and P. Boguslawski, Phys. Rev. B 83, 205205 (2011).

${ }^{25}$ Q. Yan, A. Janotti, M. Scheffler, and C. G. van de Walle, Appl. Phys. Lett. 100, 142110 (2012).

${ }^{26}$ J. Kudrnovský, I. Turek, V. Drchal, F. Máca, P. Weinberger, and P. Bruno, Phys. Rev. B 69, 115208 (2004); F. Máca, J. Kudrnovský, V. Drchal, and G. Bouzerar, Appl. Phys. Lett. 92, 212503 (2008).

${ }^{27}$ H. Jin, Y. Dai, B. B. Huang, and M.-H. Whangbo, Appl. Phys. Lett. 94, 162505 (2009).

${ }^{28}$ S. K. Nayak, M. E. Gruner, S. Sakong, S. Sil, P. Kratzer, S. N. Behera, and P. Entel, Phys. Rev. B 86, 054441 (2012).

${ }^{29}$ C. G. van de Walle and J. Neugebauer, J. Appl. Phys. 95, 3851 (2004).

${ }^{30}$ K. Laaksonen, M. G. Ganchenkova, and R. M. Nieminen, J. Phys.: Condens. Matter 21, 015803 (2009).

${ }^{31}$ F. Tuomisto, V. Ranki, D. C. Look, and G. C. Farlow, Phys. Rev. B 76, 165207 (2007). 